\title{
Full Width at Half Maximum
}

National Cancer Institute

\section{Source}

National Cancer Institute. Full Width at Half Maximum. NCI Thesaurus. Code C94903.

A measure of resolution used when a system's response y (such as image-displayed activity concentration) to an ideal infinitely narrow spatially distributed input shows a width in its values surrounding a maximum when plotted ag ainst a locating variable $\mathrm{x}$ (such as distance relative to this input location). 